\title{
Crystallization Kinetics of Poly(butylene 2,6-naphthalate) and Its Copolyesters
}

\author{
Sang Cheol LeE, ${ }^{\dagger}$ Kwan Han Yoon, and Jae Hoon Kim \\ Department of Polymer Science and Engineering, Kumoh National University of Technology, \\ Kumi, Kyungbuk 730-701, Korea
}

(Received November 9, 1995)

\begin{abstract}
The crystallization kinetics of poly(butylene 2,6-naphthalate) and its copolyesters has been investigated by differential scanning calorimetry at large undercoolings. Wide angle X-ray diffraction patterns show that the copolymers crystallize purely in crystalline phase of poly(butylene 2,6-naphthalate). In contrast to the typical crystallization behavior of copolymer, the crystallization isotherms follow the Avrami equation up to high degree of conversion, and the Avrami exponents are mostly close to 2 , independent of copolymer composition and crystallization temperature. The analysis of kinetic data with a modified Lauritzen-Hoffman equation indicates that the product of the lateral surface free energy and the fold one $\left(\sigma \sigma_{\mathrm{e}}\right)$ is $1410 \pm 100 \mathrm{erg}^{2} \mathrm{~cm}^{-4}$ and this value is not dependent on copolymer composition. The surface free energies and the work of chain folding are also discussed in comparison with those of poly(butylene terephthalate) and poly(ethylene terephthalate).

KEY WORDS Poly(butylene 2,6-naphthalate) / Large Undercooling / Copolyesters / Crystallization Rate / Surface Free Energies / Work of Chain Folding /
\end{abstract}

The temperature dependence of the crystallization rate for polymeric systems has been understood based on a classical nucleation-and-growth process. ${ }^{1,2}$ In the region of melting temperature, the growth rate is rapid compared to the nucleation rate so that the crystallization rate is governed by nucleation process and hence depends strongly on the crystallization temperature. At lower crystallization temperature, i.e., large undercoolings, the growth process, related to the transport of crystallization units onto the crystal growth surface, becomes important. Over a wide range of crystallization temperature, the crystallization kinetic data of many polymers have been analyzed with the Turnbull-Fisher equation, ${ }^{3}$ formulated in terms of nucleation and growth rates. This equation has been modified by Lauritzen and Hoffman using a molecular model, and successfully applied to a large number of homopolymers. ${ }^{4-8}$

For copolymers where one kind of component units can crystallize and the other units remain in the noncrystalline phase, there are two characteristic crystallization behaviors in contrast to homopolymer crystallization. First, the isotherms change with the crystallization temperature and do not follow the Avrami equation. Second, a noncrystallizable component strongly reduces the crystallization rate. These phenomena have been understood based on a kinetic theory for random copolymers proposed by Gornick and Mandelkern. ${ }^{9}$ According to this theory, the addition of a noncrystallizable component significantly attenuates the nucleation rate, and the nucleation rate decreases with the conversion of crystallization. Recently, Alamo and Mandel$\operatorname{kern}^{10}$ reported that several ethylene copolymers show the above two characteristics of copolymer crystallization at $25-60^{\circ}$ undercoolings.

In this study, the crystallization kinetics of poly(butylene 2,6-naphthalate)(PBN) and three poly(butylene 2,6-naphthalate-co-ethylene 2,6-naphthalate)

\footnotetext{
t To whom all correspondence should be addressed.
}

$(\mathrm{C}(\mathrm{BN}-\mathrm{EN}))$ copolyesters is investigated by differential scanning calorimetry (DSC), at large undercoolings. The objectives of this study are to investigate the characteristic phenomena of copolymer crystallization at large undercoolings ( $74-90^{\circ}$ undercoolings), and to determine the molecular parameters governing the crystallization process of these polyesters.

\section{EXPERIMENTAL}

PBN was prepared from dimethyl naphthalate and butylene glycol by melt polycondensation in the presence of titanium isopropoxide catalyst, which was described in detail elsewhere. ${ }^{11}$ Similarly, C(BN-EN) copolyesters were synthesized by the reaction of dimethyl naphthalate, butylene glycol, and ethylene glycol.

Inherent viscosities of the polyesters prepared were measured at a polymer concentration of $0.5 \mathrm{~g} \mathrm{dl}^{-1}$ in a mixed solvent of phenol-o-dichlorobenzene $(6: 4, \mathrm{v} / \mathrm{v})$ at $35^{\circ} \mathrm{C}$. Copolymer composition and sequence distribution were analyzed from ${ }^{1} \mathrm{H}$ and ${ }^{13} \mathrm{C}$ NMR spectra of its solution in a mixed solvent of trifluoroacetic acid- $\mathrm{CDCl}_{3}$ $(7: 3, v / v)$, using a Bruker AMX-500 FT-NMR spectrometer, respectively. The sequence distributions were found to be random. ${ }^{12}$ The copolymer composition and other properties are listed in Table I. Wide angle Xray scattering measurement was performed to investigate the crystalline structure of $\mathrm{PBN}$ and its copolyesters, using Rigaku X-ray diffractometer with nickelfiltered $\mathrm{Cu}-K_{\alpha}$ radiation.

The crystallization rate was measured by Du Pont 910 differential scanning calorimeter equipped with a mechanical cooling accessory. Samples were first melted at $300^{\circ} \mathrm{C}$ for $5 \mathrm{~min}$, then cooled at the rate of $70^{\circ} \mathrm{C} \mathrm{min}^{-1}$ to the crystallization temperature $\left(T_{\mathrm{c}}\right)$, and isothermally maintained at $T_{\mathrm{c}}$ unit no exothermic heat was observed. The heat of crystallization was recorded as a function of time at $T_{\mathrm{c}}$. Typically, the crystallization isotherms for PBN are shown in Figure 1. From the crystallization 
Table I. Characteristics of PBN and its copolyesters

\begin{tabular}{|c|c|c|c|}
\hline \multirow{2}{*}{ Sample } & $\begin{array}{l}\text { Copolymer } \\
\text { composition }\end{array}$ & $\begin{array}{l}\text { Inherent } \\
\text { viscosity }\end{array}$ & $T_{\mathrm{g}}^{\mathrm{a}}$ \\
\hline & $\begin{array}{l}\text { mol\% of ethylene } \\
2,6 \text {-naphthalate }\end{array}$ & & ${ }^{\circ} \mathrm{C}$ \\
\hline $\mathrm{PBN}$ & 0 & 0.98 & 52 \\
\hline C(BN-EN3) & 3.0 & 0.97 & 55 \\
\hline $\mathrm{C}(\mathrm{BN}-\mathrm{EN} 7)$ & 6.7 & 1.09 & 58 \\
\hline C(BN-EN13) & 12.9 & 0.68 & 63 \\
\hline
\end{tabular}

a Glass transition temperature $\left(T_{\mathrm{g}}\right)$ measured by DSC for the samples quenched into liquid nitrogen from the melt.

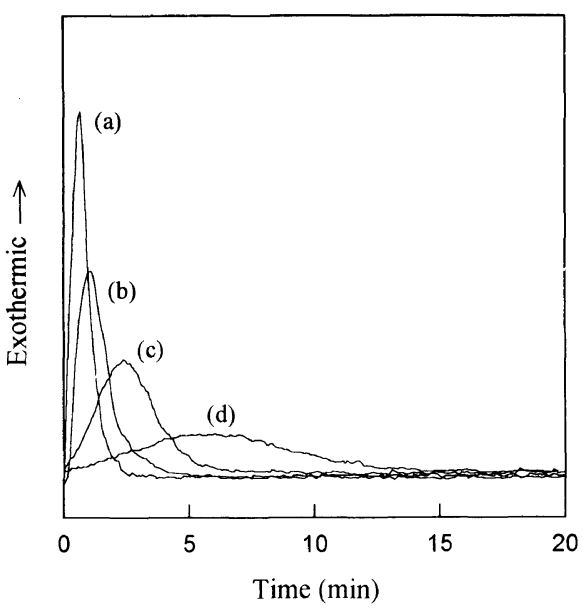

Figure 1. Crystallization isotherms for $\mathrm{PBN}$ at (a) $205.4^{\circ} \mathrm{C}$, (b) $210.5^{\circ} \mathrm{C}$, (c) $215.3^{\circ} \mathrm{C}$, and (d) $220.1^{\circ} \mathrm{C}$.

isotherms, the weight fraction $\theta_{t}$ of material crystallized after time $t$ was determined by the relation

$$
\theta_{t}=\frac{\int_{0}^{t}(\mathrm{~d} H / \mathrm{d} \tau) \mathrm{d} \tau}{\int_{0}^{\infty}(\mathrm{d} H / \mathrm{d} \tau) \mathrm{d} \tau}
$$

where $\mathrm{d} H / \mathrm{d} \tau$ is the rate of heat evolution.

\section{RESULTS AND DISCUSSION}

Figure 2 shows the wide angle $\mathrm{X}$-ray diffraction patterns for the samples crystallized isothermally at $200^{\circ} \mathrm{C}$ for $24 \mathrm{~h}$ after cooling from the melt. PBN shows the crystal diffractions reported by Watanabe, ${ }^{13}$ which are consistent with the triclinic unit cell, the lattice constants being $a=4.87, b=6.22, c=14.36 \AA, \alpha=100.78$, $\beta=126.90$, and $\gamma=97.93^{\circ}$. The diffraction peaks of the copolyesters are broader than those of PBN, caused by the reduction of crystal size. In Figure 3, the interplanar spacings $\left(d_{h k l}\right)$ calculated using the Bragg equation are plotted against the copolymer composition. The $d$-values remain almost unchanged for any of the Miller planes. And also, there is no diffraction evidence corresponding to the poly(ethylene 2,6-naphthalate) (PEN) crystal. This result represents that the copolyesters crystallize purely in the crystalline phase of PBN without the formation of isomorphic cocrystal or PEN crystal.

The crystallization isotherms were analyzed with the Avrami equation ${ }^{14,15}$

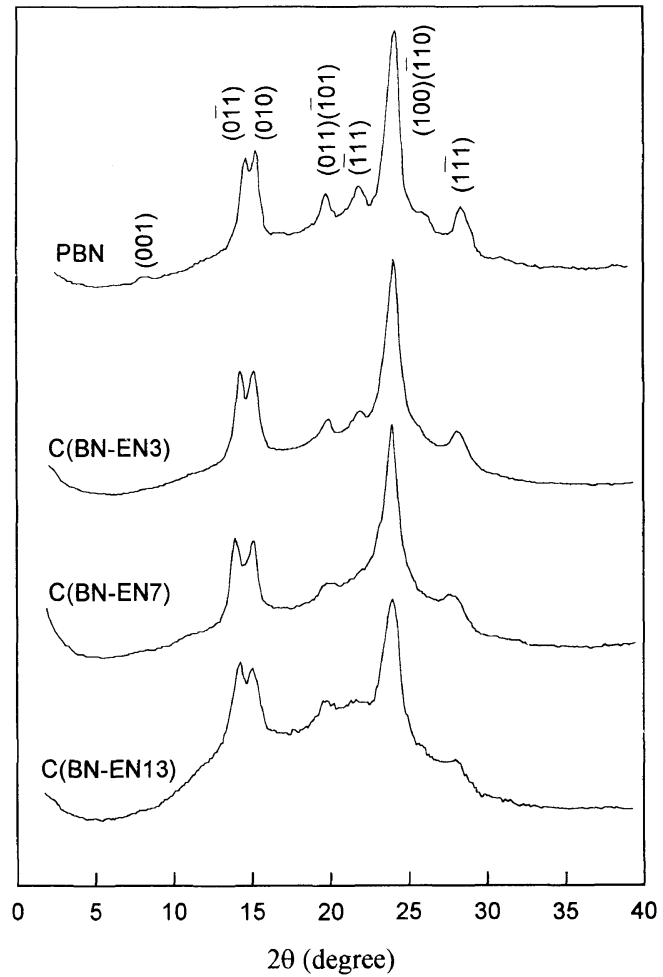

Figure 2. Wide angle $\mathrm{X}$-ray diffraction patterns for $\mathrm{PBN}$ and its copolyesters.

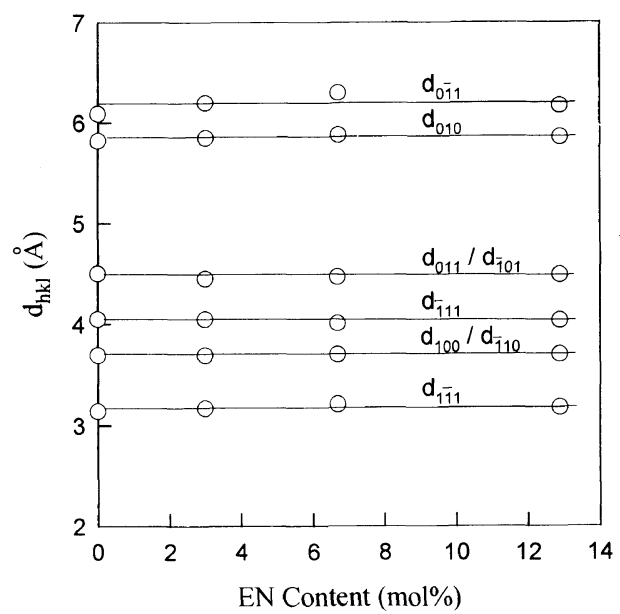

Figure 3. Plot of interplanar spacings against copolymer composition.

$$
\log \left[-\ln \left(1-\theta_{t}\right)\right]=n \log t+\log K
$$

where $K$ is the overall kinetic rate constant which depends on the rate of nucleation and growth, and $n$, so called Avrami exponent, is a parameter that depends on the nature of the primary nucleation and the geometry of growing crystals. The values of $K$ and $n$ can be determined from the intercept and the slope of a straight line in plot of $\log \left[-\ln \left(1-\theta_{t}\right)\right]$ against $\log t$. The crystallization half-time $t_{1 / 2}$, which is defined as the time at $\theta_{t}=1 / 2$, can also be calculated by using the relation

$$
t_{1 / 2}=\left(\frac{\ln 2}{K}\right)^{1 / n}
$$

As shown in Figure 4, the linear relationships between $\log \left[\left(-\ln \left(1-\theta_{t}\right)\right]\right.$ and $\log t$ for a large part of crystal- 

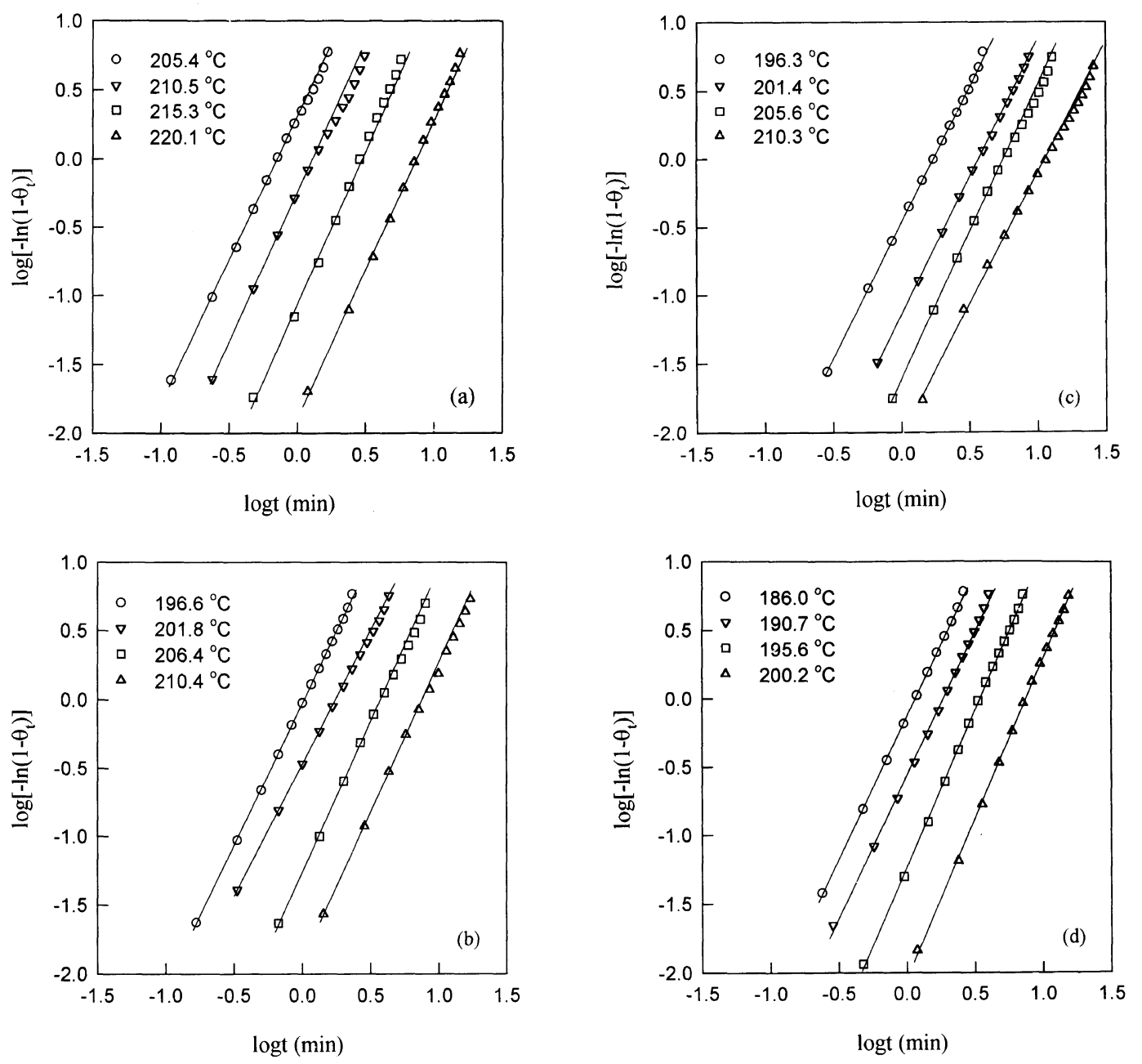

Figure 4. Avrami plots for (a) PBN, (b) C(BN-EN3), (c) C(BN-EN7), and (d) C(BN-EN13).

lization process indicate that the crystallization kinetics for all of PBN and its copolyesters follows the Avrami equation up to high degree of conversion. In general, the crystallization isotherms of copolymers deviate from the Avrami-type behavior, because the molten phase becomes enriched in the noncrystallizable component as crystallization progresses. ${ }^{9}$ However, the experimental data for the copolyesters in this study satisfy the Avrami equation, and the Avrami exponents listed in Table II are almost close to 2 , independent of crystallization temperature and copolymer composition. This means that the nucleation type and the crystal shape of the PBN homopolymer is similar to those of the copolyesters over the range of crystallization temperature investigated, and also that the isotherm shape does not change with the crystallization temperature. This result may be attributed to the fact that the nucleation rates of the copolyesters are almost constant as crystallization progresses because the Gibbs free energy difference between crystal and melt, driving force for crystallization, is much great at large undercoolings. ${ }^{9}$

Figure 5 shows that the crystallization half-time increases with the increment of the content of noncrystallizable component at a fixed crystallization temperature. This is consistent with the typical phenomenon of copolymer crystallization that the noncrystallizable component reduces the crystallization rate.

The overall crystallization rate for a copolymer $G$ can
Table II. Values of $n$ and $t_{1 / 2}$ at various $T_{\mathrm{c}}$ for $\mathrm{PBN}$ and its copolyesters

\begin{tabular}{|c|c|c|c|}
\hline \multirow{2}{*}{ Sample } & $T_{\mathrm{c}}$ & \multirow{2}{*}{$n$} & \multirow{2}{*}{$\frac{t_{1 / 2}}{\min }$} \\
\hline & ${ }^{\circ} \mathrm{C}$ & & \\
\hline \multirow[t]{4}{*}{$\mathrm{PBN}$} & 205.4 & 2.10 & 0.60 \\
\hline & 210.5 & 2.19 & 1.09 \\
\hline & 215.3 & 2.24 & 2.53 \\
\hline & 220.1 & 2.18 & 6.28 \\
\hline \multirow[t]{4}{*}{$\mathrm{C}(\mathrm{BN}-\mathrm{EN} 3)$} & 196.6 & 2.07 & 0.86 \\
\hline & 201.8 & 1.93 & 1.45 \\
\hline & 206.4 & 2.20 & 3.16 \\
\hline & 210.4 & 2.18 & 6.34 \\
\hline \multirow[t]{4}{*}{$\mathrm{C}(\mathrm{BN}-\mathrm{EN} 7)$} & 196.3 & 1.99 & 1.43 \\
\hline & 201.4 & 2.02 & 3.06 \\
\hline & 205.6 & 2.15 & 4.72 \\
\hline & 210.3 & 1.93 & 9.33 \\
\hline \multirow[t]{4}{*}{$\mathrm{C}(\mathrm{BN}-\mathrm{EN} 13)$} & 186.0 & 2.09 & 0.97 \\
\hline & 190.7 & 2.09 & 1.56 \\
\hline & 195.6 & 2.29 & 2.94 \\
\hline & 200.2 & 2.33 & 7.09 \\
\hline
\end{tabular}

be analyzed with the Lauritzen-Hoffman expression ${ }^{5}$

$$
G=x_{\mathrm{A}} G_{0} \exp \left[\frac{-U^{*}}{R\left(T_{\mathrm{c}}-T_{\infty}\right)}\right] \exp \left[\frac{-K_{\mathrm{f}}}{T_{\mathrm{c}} \Delta f}\right]
$$

where $x_{\mathrm{A}}$ is the mole fraction of crystallizable component A, which is introduced to account for the proportionality 


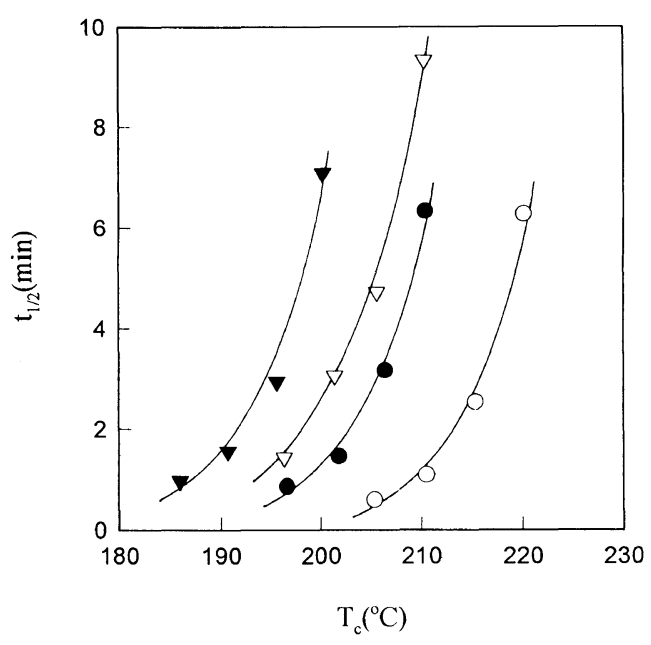

Figure 5. Plot of crystallization half-time against crystallization temperature for (O) PBN, (๑) C(BN-EN3), ( $\nabla) \mathrm{C}(\mathrm{BN}-\mathrm{EN} 7)$, and ( $)$ C (BN-EN13).

between the rate of nucleation and the fraction of crystallizable component, $G_{0}$ is a constant, $U^{*}$ is the activity energy for the diffusion of crystallizing units, $R$ is the gas constant, $T_{\infty}$ is the temperature at which the viscosity is infinite, $\Delta f$ is the bulk free energy of fusion per unit volume, and $K_{\mathrm{f}}$ is the nucleation factor expressed as

$$
K_{\mathrm{f}}=\frac{m b_{0} \sigma \sigma_{\mathrm{e}}}{k}
$$

where $m$ is a constant reflecting the regime of crystallization, $b_{0}$ is the thickness of a monomolecular layer, $\sigma$ is the lateral surface free energy, $\sigma_{\mathrm{e}}$ is the fold surface free energy, and $k$ is the Boltzmann constant. According to the Flory theory for a random copolymer where component A units only can crystallize, the bulk free energy of fusion per mole of repeating unit $\Delta f_{\mathrm{m}}$ can be expressed as ${ }^{16}$

$$
\Delta f_{\mathrm{m}}=\Delta f_{\mathrm{m}}{ }^{0}+R T_{\mathrm{c}} \ln x_{\mathrm{A}}
$$

where $\Delta f_{\mathrm{m}}{ }^{0}$ is the bulk free energy of fusion per mole of repeating unit for the corresponding homopolymer, and can be approximated by $\Delta f_{\mathrm{m}}{ }^{0} \simeq \Delta H_{\mathrm{m}}\left(1-T_{\mathrm{c}} / T_{\mathrm{m}}{ }^{0}\right)$. Here $\Delta H_{\mathrm{m}}$ and $T_{\mathrm{m}}{ }^{0}$ are the bulk heat of fusion per mole of repeating unit and the equilibrium melting temperature for the homopolymer, respectively. Assuming that the overall crystallization rate $G$ is directly proportional to the reciprocal of crystallization half-time $t_{1 / 2}^{-1}$ and considering eq 6 , eq 4 can be rearranged as

$$
\begin{aligned}
f\left(t_{1 / 2}^{-1}\right) & =\log \left(t_{1 / 2}^{-1}\right)-\log x_{\mathrm{A}}+\frac{U^{*}}{2.3 R\left(T_{\mathrm{c}}-T_{\infty}\right)} \\
& =\log \left(t_{1 / 2}^{-1}\right)_{0}-\frac{M K_{\mathrm{f}}}{2.3 \mathrm{~d} T_{\mathrm{c}}\left(\Delta f_{\mathrm{m}}{ }^{0}+R T_{\mathrm{c}} \ln x_{\mathrm{A}}\right)}
\end{aligned}
$$

where $d$ is the density of crystalline phase, and $M$ is the molecular weight of crystallizable repeating unit. In the analysis of the experimental data with eq 7, values of $M=270.28 \mathrm{~g} \mathrm{~mol}^{-1}, d=1.3872 \mathrm{~g} \mathrm{~cm}^{-3},{ }^{13} \Delta H_{\mathrm{m}}=7883 \mathrm{cal}$ $\mathrm{mol}^{-1},{ }^{17}$ and $T_{\mathrm{m}}{ }^{0}=294^{\circ} \mathrm{C}$ are used. The equilibrium melting temperature for PBN was determined by using the method of Hoffman and Weeks, ${ }^{18}$ shown in Figure

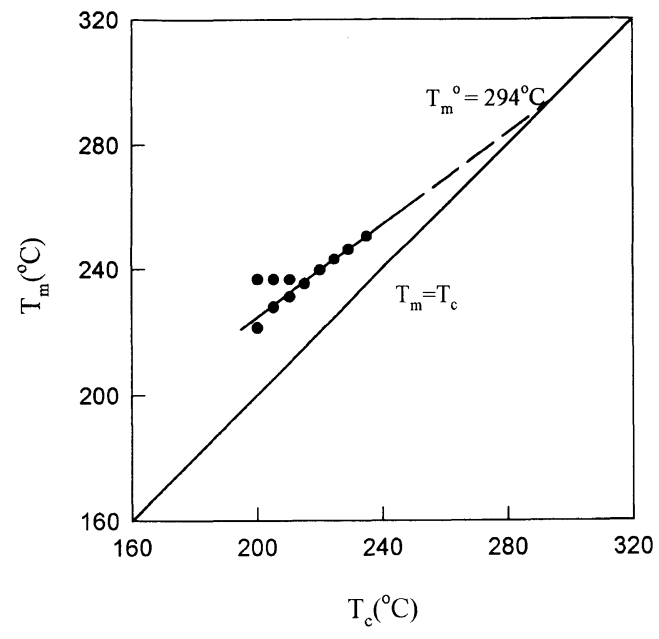

Figure 6. Hoffman-Weeks plot for PBN.

6. The samples crystallized isothermally below $215^{\circ} \mathrm{C}$ exhibit double melting peaks of PBN crystal for a mechanism based on melting, recrystallization, and subsequent remelting. Usually, the diffusion parameters are $U^{*}=1500 \mathrm{cal} \mathrm{mol}^{-1}$ and $T_{\infty}=T_{\mathrm{g}}-30 \mathrm{~K}^{4}{ }^{4}$ On the other hand, values of $U^{*}=4120 \mathrm{cal} \mathrm{mol}^{-1}$ and $T_{\infty}=T_{\mathrm{g}}$ $51.6 \mathrm{~K}$, found by Williams, Landel, and Ferry (WLF), ${ }^{19}$ have been also used. In this study, the data were analyzed with both diffusion parameters. In Figure $7, f\left(t_{1 / 2}^{-1}\right)$ was plotted against $M /\left[\mathrm{d} T_{\mathrm{c}}\left(\Delta f_{\mathrm{m}}{ }^{0}+R T_{\mathrm{c}} \ln x_{\mathrm{A}}\right)\right]$. In the plot using $U^{*}=1500 \mathrm{cal} \mathrm{mol}^{-1}$ and $T_{\infty}=T_{\mathrm{g}}-30 \mathrm{~K}$, the data of copolyesters keep apart from those of PBN. However, all data points, analyzed with WLF diffusion parameters, almost fall on the same line. This represents that eq 7, applied with WLF diffusion parameters, satisfactorily governs the crystallization process, and also that the product $\sigma \sigma_{\mathrm{e}}$, which can be determined by the slope of the line, is not dependent on copolymer composition. If $\sigma$ is independent of copolymer composition, then $\sigma_{\mathrm{e}}$ must also be. ${ }^{10}$ Hence, it is considered that $\sigma$ and $\sigma_{\mathrm{e}}$ values of the copolyesters are equal to those of the pure PBN homopolymer, respectively. The lateral surface free energy $\sigma$ is related with the heat of fusion $\Delta H$ as the following Thomas-Stavely relation ${ }^{20}$

$$
\sigma=\alpha \Delta H\left(a_{0} b_{0}\right)^{1 / 2}
$$

where $\alpha$ is an empirical constant and $a_{0}$ is the chain stem width. The heat of fusion of copolymer can be considered as the sum of the heat of fusion of homopolymer and the heat of mixing of the pure melt with a noncrystallizable component. There is no considerable difference in the heat of fusion between homopolymer and copolymer, because the heat of mixing is negligible in comparison with the heat of fusion of homopolymer. Hence, if we accept the above relation, $\sigma$ value of copolymer is almost equal to that of homopolymer. Fold surface free energy $\sigma_{\mathrm{e}}$ is composed of the surface enthalpic and entropic terms. The surface entropy depends on the number of units within a fold. The average fold length of the copolymer is longer than that of homopolymer because of the presence of noncrystallizable component in copolymer. It is expected that the surface entropy of copolymer is larger than that of homopolymer, resulting in the reduction of surface free energy. On the other 

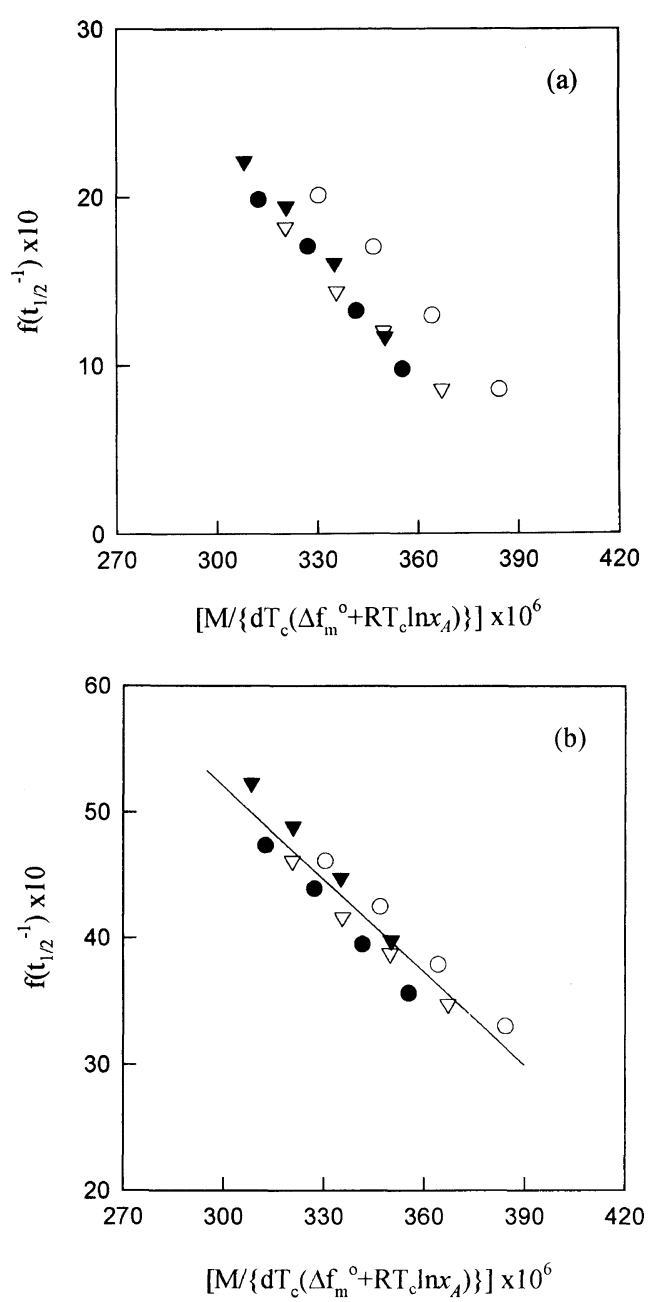

Figure 7. Plots of $f\left(t_{1 / 2}^{-1}\right)$ against $\mathrm{M} /\left[\mathrm{d} T_{\mathrm{c}}\left(\Delta f_{\mathrm{m}}{ }^{0}+R T_{\mathrm{c}} \ln x_{\mathrm{A}}\right)\right]$ using (a) $U^{*}=1500 \mathrm{cal} \mathrm{mol}^{-1}, \quad T_{\infty}=T_{\mathrm{g}}-30 \mathrm{~K}$, and (b) $U^{*}=4120 \mathrm{cal} \mathrm{mol}^{-1}$, $T_{\infty}=T_{\mathrm{g}}-51.6 \mathrm{~K}$, for (O) PBN, $(\bigcirc) \mathrm{C}(\mathrm{BN}-\mathrm{EN} 3),(\nabla) \mathrm{C}(\mathrm{BN}-\mathrm{EN} 7)$, and

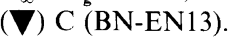

hand, the incorporation of noncrystallizable units in a copolymer probably causes the increase of the enthalpy. The constancy of $\sigma_{\mathrm{e}}$ with copolymer composition should be ascribed to the fact that the enthalpic contribution cancels the entropic one to the fold surface free energy.

In order to calculate the product $\sigma \sigma_{\mathrm{e}}$ from the line slope in Figure 5(b), $m$ value, which depends on the regime of crystallization ( $m=4$ for regimes I and III, $m=2$ for regime II), must be previously determined. Lauritzen ${ }^{21}$ proposed the $Z$ test to determine whether the crystallization is in regime I or regime II. $Z$ is defined as

$$
Z \simeq 10^{3}\left(\frac{L}{2 a_{0}}\right)^{2} \exp \left[\frac{-X}{T_{\mathrm{c}} \Delta f}\right]
$$

where $L$ is the length of the substrate lamella. The criterion of the $Z$ test for each regime is

$$
\begin{aligned}
& X=K_{\mathrm{f}} \quad \text { for test of region I, } \quad Z \leq 0.01 \\
& X=2 K_{\mathrm{f}} \quad \text { for test of regime II }, \quad Z \geq 1
\end{aligned}
$$

With eq 9,10 , and the known value of $K_{\mathrm{f}}$ obtained from the line slope, it is possible to estimate the range of $L$ values and decide whether these $L$ values are reasonable in each regime or not. The crystal structure of $\mathrm{PBN}$ is known, ${ }^{13}$ but there is no information on the fold direction. Therefore, it is assumed that chain folding occurs along (010) planes, analogous to PBT, ${ }^{22}$ then $a_{0}$ and $b_{0}$ values are estimated to be $3.89 \AA$ and $5.79 \AA$, respectively. The $Z$ test for regime I shows that $L$ should be less than $150-1300 \AA$ for all samples. This is realistic. Similar test for regime II requires $L$ to be greater than $920-67000 \mu \mathrm{m}$, which is unrealistic. In spite of the result of above $Z$ test, we can not conclude confidently that the crystallization within the $T_{\mathrm{c}}$ range investigated belongs to regime I, because of the possibility for crystallizing in regime III. Although it is difficult to determine whether the crystallization follows regime I or regime III only with the data obtained in this study, we can preclude regime II, and then decide $m=4$.

Taking into account the empirical error with $T_{\mathrm{m}}{ }^{0}=$ $294 \pm 3^{\circ} \mathrm{C}$, the product $\sigma \sigma_{\mathrm{e}}$ is found to be $1410 \pm$ $100 \mathrm{erg}^{2} \mathrm{~cm}^{-4}$. The surface free energies $\sigma, \sigma_{\mathrm{e}}$ and the work of chain folding $q$ can be determined using eq 8 and the following relations

$$
\begin{aligned}
& \sigma_{\mathrm{e}}=\frac{\sigma \sigma_{\mathrm{e}}}{\sigma} \\
& q=2 a_{0} b_{0} \sigma_{\mathrm{e}}
\end{aligned}
$$

The $\alpha$ value in eq 8 is usually assumed to be 0.1 for polyolefines and 0.25 for polyesters with high melting temperature. ${ }^{23}$ However, it is difficult to decide a realistic value for $\alpha$. Runt $e t a l{ }^{22}$ reported that the $\sigma_{\mathrm{e}}$ and $q$ values calculated with $\alpha=0.1$ are more reasonable for PBT. Recalling that PBN has the same chemical structure as PBT except for having naphthalene ring instead of benzene ring, it is expected that the $\alpha$ value for $\mathrm{PBN}$ is close to that for PBT. Estimating with $\alpha=0.1$ and $\Delta H=169 \mathrm{~J} \mathrm{~cm}^{-3}$ leads to $\sigma=8.0 \mathrm{erg} \mathrm{cm}^{-2}, \sigma_{\mathrm{e}}=176 \pm 14$ erg cm${ }^{-2}$, and $q=11 \pm 1 \mathrm{kcal} \mathrm{mol}^{-1}$. The $\sigma$ value is close to those reported for PBT $\left(8.8 \mathrm{erg} \mathrm{cm}^{-2}\right)$ and PET (10.6 erg cm $\left.{ }^{-2}\right){ }^{22}$ But latter two values are larger than those for PBT $\left(\sigma_{\mathrm{e}}=57-75 \mathrm{erg} \mathrm{cm}^{-2}, q=3-5 \mathrm{kcal} \mathrm{mol}^{-1}\right)$, and are comparable to those for PET $\left(\sigma_{\mathrm{e}}=\sim 140 \mathrm{erg} \mathrm{cm}^{-2}\right.$, $\left.q=\sim 10 \mathrm{kcal} \mathrm{mol}^{-1}\right){ }^{22}$ This result may be caused by the fact that naphthalene unit onto PBN makes PBN chains stiffer than PBT chains, and hence chain mobility of PBN necessary to form chain folds is alike to that of PET, having ethylene unit instead of butylene one.

\section{CONCLUSIONS}

The crystallization kinetics of PBN and its copolyesters has been examined using DSC at large undercoolings. The crystallization isotherms follow the Avrami equation up to high degree of conversion, and their shapes are superposable, in contrast to the typical crystallization behavior of copolymer. On the other hand, the crystallization rate decreases with the increment of the content of noncrystallizable component.

The overall crystallization rate is analyzed with a modified Lauritzen-Hoffman equation, and this equation, applied with WLF diffusion parameters, satisfactorily governs the crystallization process. The lateral surface free energy $\sigma$, fold surface free energy $\sigma_{\mathrm{e}}$, and work of chain folding $q$ are determined from LauritzenHoffman equation and Thomas-Stavely relation. The values of $\sigma, \sigma_{\mathrm{e}}$, and $q$ are $8.0 \mathrm{erg} \mathrm{cm}^{-2}, 176 \pm 14 \mathrm{erg} \mathrm{cm}^{-2}$, and $11 \pm 1 \mathrm{kcal} \mathrm{mol}^{-1}$, respectively, and these values are 
independent of copolymer composition.

\section{REFERENCES}

1. L. Mandelkern, "Crystallization of Polymers," MaGraw Hill, New York, N.Y., 1964.

2. B. Wunderlich, "Macromolecular Physics," Vol. 2, Academic Press, New York, N.Y., 1976

3. D. Turnbull and J. C. Fisher, J. Chem. Phys., 17, 71 (1949).

4. J. D. Hoffman, G. T. Davis, and J. I. Lauritzen, Jr., "Threatise on Solid-State Chemistry," Vol. 3, N. D. Hannay, Ed., Plenum Press, New York, N.Y., 1976.

5. J. I. Lauritzen, Jr. and J. D. Hoffman, J. Appl. Phys., 44, 4340 (1973).

6. J. D. Hoffman, Polymer, 24, 3 (1983).

7. C. J. Ong and F. P. Price, J. Polym. Sci., Polym. Symp., 63, 59 (1978).

8. P. J. Phillips, G. J. Rensch, and K. D. Tayor, J. Polym. Sci., Polym. Phys., 25, 1725 (1978).

9. F. Gornick and L. Mandelkern, J. Appl. Phys., 33, 907 (1962).
10. R. G. Alamo and L. Mandelkern, Macromolecules, 24, 6480 (1991).

11. K. H. Yoon, S. C. Lee, and O. O. Park, Polym. J., 26, 816 (1994).

12. S. C. Lee and K. H. Yoon, in preparation.

13. H. Watanabe, Kobunshi Ronbunshu, 33, 229 (1976).

14. M. Avrami, J. Chem. Phys., 7, 1103 (1939); ibid., 8, 212 (1940); ibid., 9, 177 (1941).

15. L. Mandelkern, F. A. Quinn, Jr., and P. J. Flory, J. Appl. Phys., 25, 830 (1954); ibid., 26, 443 (1955).

16. P. J. Flory, J. Chem. Phys., 17, 223 (1949).

17. D. W. Van Krevelen, "Properties of Polymers," Elsevier Scientific, Amsterdam, 1990, Chapter 5.

18. J. D. Hoffman and J. J. Weeks, J. Chem. Phys., 42, 4301 (1965).

19. M. L. Williams, R. F. Landel, and J. D. Ferry, J. Am. Chem. Soc., 77, 3701 (1975).

20. D. G. Thomas and L. A. K. Staveley, J. Chem. Soc., 4569 (1952).

21. J. I. Launitzen, Jr., J. Appl. Phys., 44, 4353 (1973).

22. J. Runt, D. M. Miley, X. Zhang, K. P. Gallagher, K. McFeaters, and J. Fishburn, Macromolecules, 25, 1929 (1992).

23. D. B. Roitman, H. Marand, R. L. Miller, and J. D. Hoffman, J. Phys. Chem., 93, 6919 (1989). 